\title{
Dataset: Longitudinal personal thermal comfort preference data in the wild
}

\author{
Matias Quintana* \\ matias@u.nus.edu \\ National University of Singapore
}

\author{
Mahmoud Abdelrahman* \\ mahmoud@u.nus.edu \\ National University of Singapore
}

\author{
Mario Frei \\ mario.frei@nus.edu.sg \\ National University of Singapore
}

\author{
Federico Tartarini \\ federico.tartarini@bears-berkeley.sg \\ Berkeley Education Alliance for \\ Research in Singapore
}

\author{
Clayton Miller ${ }^{\dagger}$ \\ clayton@nus.edu.sg \\ National University of Singapore
}

\begin{abstract}
Thermal comfort affects the well-being, productivity, and overall satisfaction of building occupants. However, due to economical and practical limitations, the number of longitudinal studies that have been conducted is limited, and only a few of these studies have shared their data publicly. Longitudinal datasets collected indoors are a valuable resource to better understand how people perceive their environment. Moreover, they provide a more realistic scenario to those conducted in thermal chambers. Our objective was to share publicly a longitudinal dataset comprising data collected over a 4week long experiment. A total of 17 participants completed thermal preferences surveys which accounted for a total of approximately 1400 unique responses across indoor and outdoor 17 spaces. For the whole duration of the study, we monitored environmental variables (e.g., temperature and relative humidity) throughout 3 buildings. Participants completed comfort surveys from the screen of their smartwatches using an open-source application named Cozie. Their indoor location was continuously monitored using a customdesigned smartphone application. Location data were used to time and spatially align environmental measurements to thermal preference responses provided by the participants. Background information of participants, such as physical characteristics and personality traits (satisfaction with life scale, highly sensitive person scale, the Big Five personality traits), was collected using an on-boarding survey administered at the beginning of the experiment. The dataset is available at https://zenodo.org/record/5502441\#.YT7xyaARUTs.
\end{abstract}

\section{CCS CONCEPTS}

- Human-centered computing $\rightarrow$ Ubiquitous and mobile devices; • Social and professional topics $\rightarrow$ User characteristics; • Hardware $\rightarrow$ Sensor applications and deployments.

\footnotetext{
*Equal contribution.

$\dagger$ Corresponding author.
}

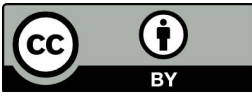

This work is licensed under a Creative Commons Attribution International 4.0 License.

SenSys'21, November 17, 2021, Coimbra, Portugal,

(c) 2021 Copyright held by the owner/author(s). Publication rights licensed to ACM. ACM ISBN 978-1-4503-9097-2/21/11 ..\$15.00

https://doi.org/10.1145/3485730.3493693

\section{KEYWORDS}

Datasets, Thermal comfort, Smart buildings, Longitudinal experiment

ACM Reference Format:

Matias Quintana, Mahmoud Abdelrahman, Mario Frei, Federico Tartarini, and Clayton Miller. 2021. Dataset: Longitudinal personal thermal comfort preference data in the wild. In The 19th ACM Conference on Embedded Networked Sensor Systems (SenSys '21), November 15-17, 2021, Coimbra, Portugal. ACM, New York, NY, USA, 4 pages. https://doi.org/10.1145/3485730. 3493693

\section{INTRODUCTION}

Understanding how people perceive their thermal environment helps researchers and practitioners to optimize the operation and design of heating, ventilation, and air-conditioning (HVAC) systems. To better predict how people perceive their environment thermal comfort researchers are moving towards creating personalized comfort models. This transition is facilitated by the proliferation of Internet-of-Things (IoT) sensors and wearable devices which allow researchers to non-intrusively measure and collect a wide array of data, e.g, environmental factors, and physiological measurements. These data can then be used to train and test a variety of comfort models. However, conducting longitudinal studies remains to date a difficult task due to economical and practical constraints. For this reason, the great majority of publicly available thermal comfort datasets are either from cross-sectional studies [4] or were conducted in thermal chambers. Existing longitudinal datasets either cover a short time window of 2-weeks [6] or use sensors that are not feasible to deploy in a field study [10]. Therefore, the objective of this work is to share a longitudinal field study where off-the-shelf, non-intrusive sensors are used to capture a wide array of data for a period of 4-weeks from 17 participants alongside their right-hereright-now thermal preference. While background information were collected using on-boarding questionnaires.

\section{METHODOLOGY}

The data collection experiment took place at the National University of Singapore. This location is characterized by a tropical climate (Af - Köppen-Geiger climate classification). Participants completed a one-time on-boarding survey, with multiple questionnaires, used to capture background information at the beginning of the experiment. The on-boarding survey comprised the following questions: 
(1) Physical characteristics: Weight, height, and shoulder circumference.

(2) Background information: Age, sex, number of years in Singapore, and acclimatization-related questions. The answers were recorded numerically and the specific variable types and ranges are explained in the documentation file published in the public repository.

(3) Personality surveys: Responses to the Highly Sensitive Person Scale (HSPS) survey [2], Satisfaction With Life Scale (SWLS) survey [3], and a brief version of the Big-Five personality scale [5] are recorded in a 7-point Likert scale. These surveys are intended to capture personality traits and preferences and have been used in field experiments for health monitoring [9]. The responses for all surveys are recorded in both their raw format and their aggregated final score following each respective survey's instructions.

During the 4-week data collection period, participants were asked to complete 80 right-here-right-now surveys via the smartwatch application Cozie [7]. Each survey comprised questions about thermal preference, clothing level, metabolic rate, perceived air velocity, and location. This application has been used to collect similar preferences responses in previous studies [1, 6, 11, 13] with very little impact on the participants' activities and it can be installed for free on both Android ${ }^{1}$ and Apple $^{2}$ platforms. Participants were not required to respond at specific times of the day, as long as they completed two responses more than 15 minutes apart and while within three educational buildings. These buildings comprised of a total of 17 indoor and outdoor study spaces where environmental sensors were installed to measure the following parameters:

\subsection{Environmental and physiological measurements}

(1) Outdoor Environment: One PurpleAir-PA-II ${ }^{3}$ outdoor weather station was installed at the same floor in each building. These devices measured air temperature, relative humidity, pressure, particulate matter, and particles concentration.

(2) Indoor Environment: Due to technical limitations, different sensors were used to measure indoor environmental variables. Network-connected data loggers were used when possible (Awair Omni ${ }^{4}$ and SenSING AirSENSE ${ }^{5}$ ) given their capability to record additional environmental measurements like $\mathrm{CO}_{2}$, VOC, and PM2.5. However, UbiBot WS1-Pro ${ }^{6}$ and Building Management System (BMS) wall sensors were also used for data redundancy.

(3) Indoor Location: was monitored using a mobile application called YAK [6] which scanned for Bluetooth beacons installed throughout the three buildings.

\footnotetext{
${ }^{1}$ https://github.com/cozie-app/cozie

${ }^{2} \mathrm{https} / /$ github.com/cozie-app/cozie-apple

${ }^{3}$ https://www2.purpleair.com/collections/air-quality-sensors/products/purpleair-paii

${ }^{4}$ https://www.getawair.com/products/omni

${ }^{5} \mathrm{https} / / /$ sensing.online/

${ }^{6}$ https://www.ubibot.com/ubibot-ws1pro/
}

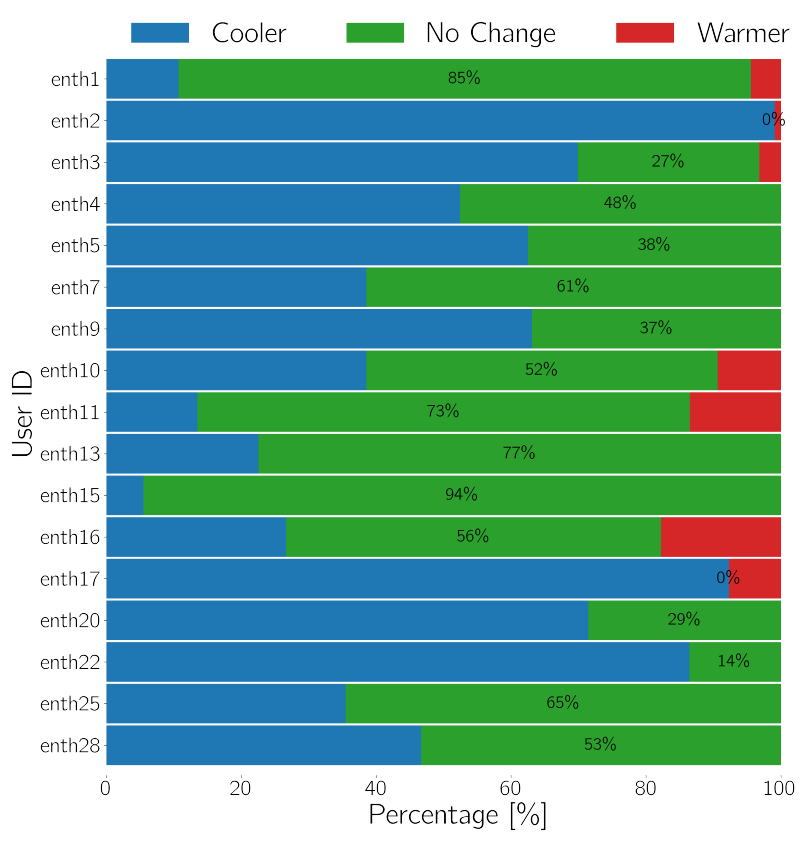

Figure 1: Thermal preference responses distribution for all participants in the dataset

(4) Physiological: Each participant was given a Fitbit Versa (v1 or v2) smartwatch. Two iButtons ${ }^{7}$, attached to the inner and outer side of the smartwatch strap, was used to measure skin and near-body temperature, respectively. The smartwatch measured heart rate and resting heart rate, as well as, if the participant was wearing it.

Location data were used to spatially and temporally align environmental readings with preferences responses from the participants. Steps to reproduce the convergence of data streams is available on our public GitHub ${ }^{8}$.

\section{RESULTS}

A total of 17 participants were recruited (10 Males and 7 Females). They provided 1403 unique responses across 17 indoor and outdoor spaces. The data collection experiment was given the code name enth and each participant was given a unique identifier consisting of the code name and an ordinal number, e.g., enth01. The distribution of thermal preference responses for all participants is shown in Figure 1 and the distribution of skin and near-body temperatures each participant experiences are shown in Figure 2.

A summary plot of all on-boarding surveys (HSPS, SWLS, and the Big-Five personality traits: "Extraversion", "Agreeableness", "Conscientiousness", "Emotional stability", and "Openness to experiences”) responses for all users is shown in Figure 3. Participants' scores for "Opennes to experiences" have more variability than the other Big-Five personality traits scores (0.31 standard deviation more on average). These results can either be used independently

\footnotetext{
${ }_{7}^{7}$ https://www.maximintegrated.com/en/products/ibutton-one-wire/dataloggers/DS1925.html

${ }^{8}$ https://github.com/buds-lab/longitudinal-personal-thermal-comfort
} 


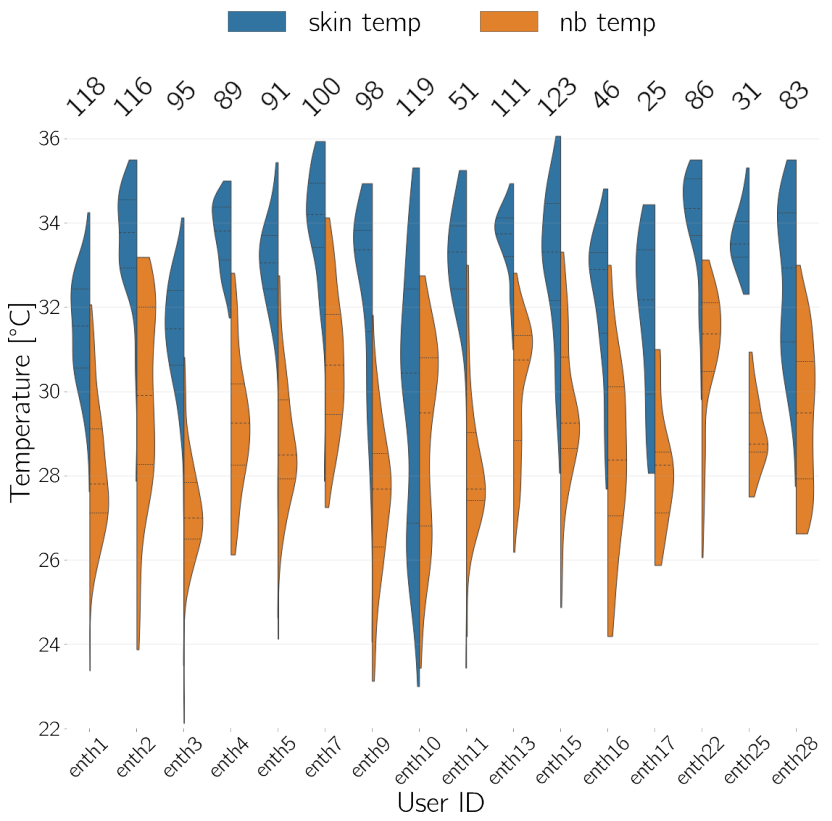

Figure 2: Wrist skin temperature (skin temp) and wrist near body temperature (nb temp) were measured when participants provided a thermal preference response. The number above each violin plot is the number of subjective responses completed by each participant. User enth20 did not log skin and near-body temperatures.

by using each trait as a model feature or as metadata for the participant, or together to find similarities among participants with unsupervised learning methods [9].

\section{DISCUSSION AND USES CASES}

Personal thermal comfort models need labeled data, collected by various people in longitudinal studies, to be trained and tested. The dataset shown here provides an average of 73 responses per participant over the span of 4 -weeks. Participants provided responses in a mix of 17 indoor and outdoor spaces within three different educational buildings and their background information was gathered through on-boarding surveys (HSPS, SWLS, and Big-Five personality trait surveys). This sets the stage for more dissemination of thermal comfort field experiments with the goal of a bigger participant pool but also a increase in background diversity of its participants. Current research applications of this work include:

Personality and thermal comfort: Current research in personalized health monitoring has shown the value of grouping users based on their responses to the Big-Five personality survey [9]. Clustering users preemptively based on their personality or preferences and finding which trait, in particular, provides the best separability, could improve on scenarios where Personal Comfort Models and Personal Comfort Systems [8] may not be feasible. However, a diverse pool of users is often required such that enough variability of preferences and personality traits are capture, i.e., health monitoring analysis is often done with 200 participants [9].

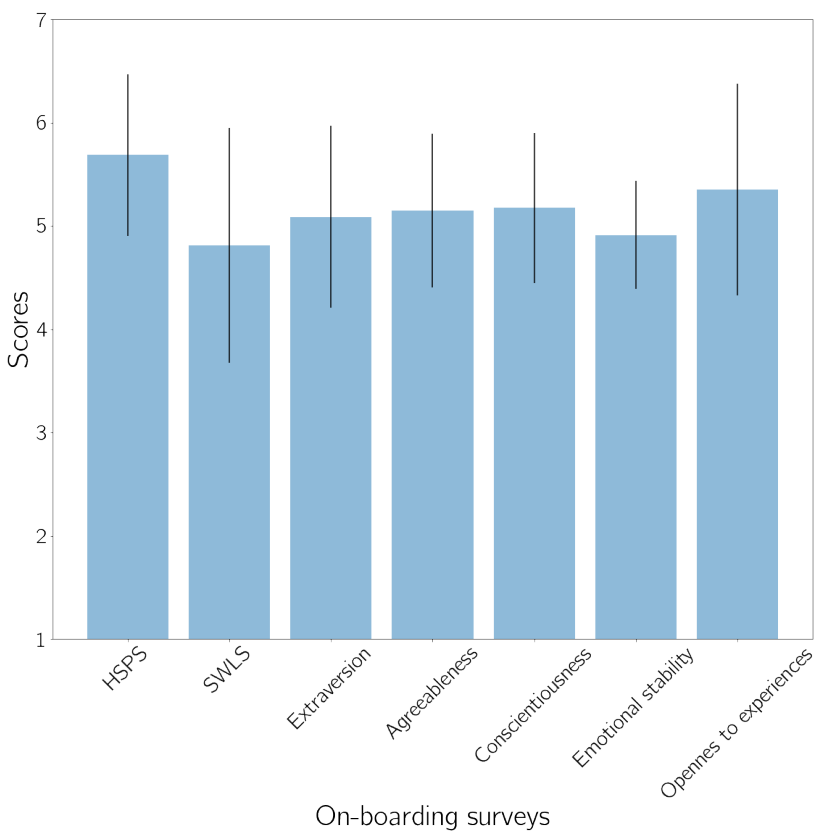

Figure 3: Summary of on-boarding survey responses for all participants. The height of the bar represent the mean of all participants' scores with their respective standard deviation bars.

Spatio-temporal thermal comfort: Contextualizing the thermal preference according to the participants' surroundings and their relationship [11] is a promising avenue to leverage spatial and temporal data for personalized workspace recommendation [1].

Building Controls: One of the goals of accurately predicting thermal preference is to correctly assess occupant's comfort when different building control strategies are chosen. However, a recent literature review on thermal comfort and building controls found that control papers fail to use a consistent criterion for thermal comfort evaluation [12]. Therefore, future control research should embrace further comprehensive evaluation and analysis of thermal comfort, which is possible with richer and diverse public datasets.

\section{ACKNOWLEDGMENTS}

We thank Ang Si Hui Pearlyn, Charis Boey Shand Yin, Chua Yun Xuan, Renee D. Christensen, Tan Charlene, Teo Yi Ting, and Muhammad Zikry Bin Sabarudin for their help during the human studies. This research was funded by the Singapore Ministry of Education (MOE) through the Tier 1 grants: Ecological Momentary Assessment (EMA) for Built Environment Research (R296000210114) and The Internet-of-Buildings (IoB) Platform - Visual Analytics for AI Technologies towards a Well and Green Built Environment (R296000214114). The Republic of Singapore's National Research Foundation (NRF) through the SinBerBEST program provided partial support for manpower for this project. 


\section{REFERENCES}

[1] Mahmoud Abdelrahman, Adrian Chong, and Clayton Miller. 2020. Build2Vec Building Representation in Vector Space. arXiv preprint arXiv:2007.00740 (2020).

[2] Elaine Nancy Aron and Arthur Aron. 1997. Sensory-Processing Sensitivity and Its Relation to Introversion and Emotionality Sensory-Processing Sensitivity and Its Relation to Introversion and Emotionality. Fournal of Personal and Social Psychology 73, 2 (1997), 345-368. https://doi.org/10.1037/0022-3514.73.2.345

[3] Ed Diener. 2006. Understanding Scores on the Satisfaction with Life Scale. (2006) 1-3.

[4] Veronika Földváry Ličina, Toby Cheung, Hui Zhang, Richard de Dear, Thomas Parkinson, Edward Arens, Chungyoon Chun, Stefano Schiavon, Maohui Luo, Gail Brager, Peixian Li, Soazig Kaam, Michael A. Adebamowo, Mary Myla Andamon, Francesco Babich, Chiheb Bouden, Hana Bukovianska, Christhina Candido, Bin Cao, Salvatore Carlucci, David K.W. Cheong, Joon-Ho Choi, Malcolm Cook, Paul Cropper, Max Deuble, Shahin Heidari, Madhavi Indraganti, Quan Jin, Hyojin Kim, Jungsoo Kim, Kyle Konis, Manoj K. Singh, Alison Kwok, Roberto Lamberts, Dennis Loveday, Jared Langevin, Sanyogita Manu, Cornelia Moosmann, Fergus Nicol, Ryozo Ooka, Nigel A. Oseland, Lorenzo Pagliano, Dušan Petráš, Rajan Rawal, Ramona Romero, Hom Bahadur Rijal, Chandra Sekhar, Marcel Schweiker Federico Tartarini, Shin-ichi Tanabe, Kwok Wai Tham, Despoina Teli, Jorn Toftum, Linda Toledo, Kazuyo Tsuzuki, Renata De Vecchi, Andreas Wagner, Zhaojun Wang, Holger Wallbaum, Lynda Webb, Liu Yang, Yingxin Zhu, Yongchao Zhai, Yufeng Zhang, and Xiang Zhou. 2018. Development of the ASHRAE Global Thermal Comfort Database II. Building and Environment 142 (sep 2018), 502-512. https://doi.org/10.1016/j.buildenv.2018.06.022

[5] Samuel D. Gosling, Peter J. Rentfrow, and William B. Swann. 2003. A very brief measure of the Big-Five personality domains. Fournal of Research in Personality 37, 6 (2003), 504-528. https://doi.org/10.1016/S0092-6566(03)00046-1
[6] Prageeth Jayathissa, Matias Quintana, Mahmoud Abdelrahman, and Clayton Miller. 2020. Humans-as-a-sensor for buildings: Intensive longitudinal indoor comfort models. Buildings 10, 174 (2020), 1-23. https://doi.org/10.3390/ buildings 10100174

[7] Prageeth Jayathissa, Matias Quintana, Tapeesh Sood, Negin Narzarian, and Clayton Miller. 2019. Is your clock-face cozie ? A smartwatch methodology for the in-situ collection of occupant comfort data. In CISBAT2019 Climate Resilient Buildings - Energy Efficiency \& Renewables in the Digital Era. Lausanne, Switzerland.

[8] Joyce Kim, Yuxun Zhou, Stefano Schiavon, Paul Raftery, and Gail Brager. 2018. Personal comfort models: Predicting individuals' thermal preference using occupant heating and cooling behavior and machine learning. Building and Environment 129, December 2017 (2018), 96-106. https://doi.org/10.1016/j.buildenv.2017.12.011

[9] Boning Li and Akane Sano. 2020. Extraction and Interpretation of Deep Autoencoder-based Temporal Features from Wearables for Forecasting Personalized Mood, Health, and Stress. Proceedings of the ACM on Interactive, Mobile, Wearable and Ubiquitous Technologies 4, 2 (2020), 1-26. https://doi.org/10.1145/3397318

[10] Shichao Liu, Stefano Schiavon, Hari Prasanna Das, Costas J Spanos, and Ming Jin. 2019. Personal thermal comfort models with wearable sensors. Building and Environment (2019), 106281. https://doi.org/10.1016/j.buildenv.2019.106281

[11] Clayton Miller, Mahmoud Abdelrahman, Adrian Chong, Filip Biljecki, Matias Quintana, Mario Frei, Michael Chew, and Daniel Wong. [n. d.]. The Internet-ofBuildings (IoB)-Digital twin convergence of wearable and IoT data with GIS/BIM. ([n. d.]).

[12] June Young Park and Zoltan Nagy. 2018. Comprehensive analysis of the relationship between thermal comfort and building control research - A data-driven literature review. Renewable and Sustainable Energy Reviews 82, July (2018), 2664-2679. https://doi.org/10.1016/j.rser.2017.09.102

[13] Pimpatsohn Sae-Zhang, Matias Quintana, and Clayton Miller. 2020. Differences in thermal comfort state transitional time among comfort preference groups. In 16th Conference of the International Society of Indoor Air Quality and Climate: Creative and Smart Solutions for Better Built Environments, Indoor Air 2020. 